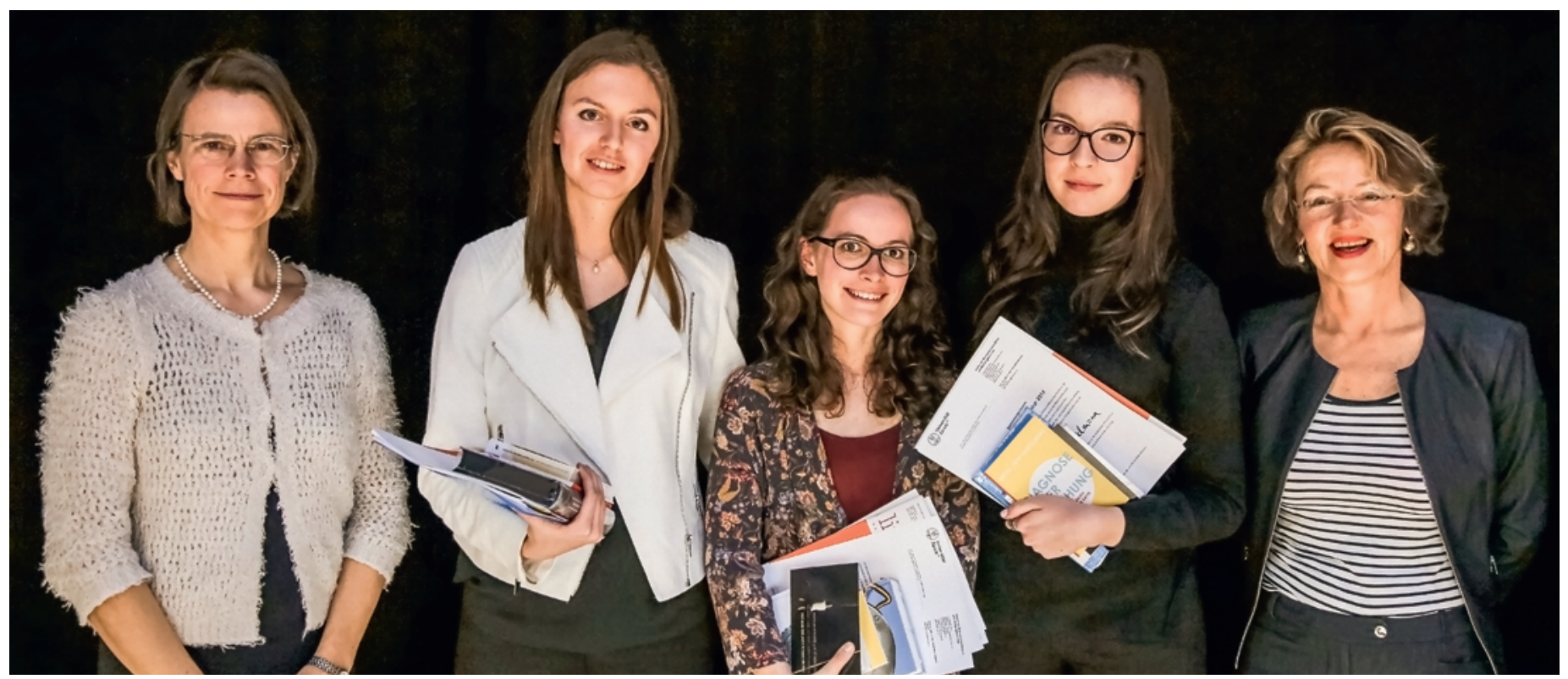

Die drei Finalistinnen des «Premio Pusterla Junior», umrahmt von den Initiantinnen des Preises (v.I.n.r.): Ethikprofessorin Nikola Biller-Andorno, Preisträgerin Selina Steiger, Gioia Epprecht, Andrina Singelmann und Hildegard Keller, Professorin für deutsche Literatur.

\title{
Die Perspektive der Patienten erhalten
}

\section{Adrian Ritter}

Freier Journalist

\begin{abstract}
Studierende der Medizin an der Universität Zürich können sich neu an einem Essay-Wettbewerb zu medizinethischen Fragen beteiligen. Im März wurde der «Premio Pusterla Junior» zum ersten Mal vergeben.
\end{abstract}

\section{Erste Vergabe des Premio Pusterla Junior}

An der Universität Zürich wird mit dem Kurs «Grundlagen der Ethik in der Medizin» bereits im ersten Studienjahr das Bewusstsein von Studierenden der Human- und Zahnmedizin für die ethischen Dimensionen der ärztlichen Tätigkeit geschärft.

Die Studierenden schreiben im Verlauf dieses Kurses einen Essay, in dem sie eine eigene Erfahrung oder ein Ereignis erzählen und ethisch reflektieren. In diesem Jahr konnten die Studierenden mit ihren Texten erstmals an einem Essay-Wettbewerb zur Vergabe des neugeschaffenen Premio Pusterla Junior teilnehmen. Drei Autorinnen qualifizierten sich für das öffentliche Finale mit Live-Jury, das am 7. März 2018 in Zürich stattfand. Die SÄZ widmet dem Preis in dieser Ausgabe zwei Beiträge und präsentiert der Leserschaft auch den Text der ersten Preisträgerin des Premio Pusterla Junior, Selina Steiger.

(UZH/SÄZ)
Allgemeine Chemie, Molekulare Zellbiologie und Anatomische Grundlagen der Medizin: Der Lehrplan des ersten Studienjahres in Medizin an der Universität Zürich ist reich befrachtet. Neben der naturwissenschaftlichen Basis des Fachs sollen die humanwissenschaftlichen Aspekte nicht zu kurz kommen - etwa im Kurs «Grundlagen der Ethik in der Medizin». Zum Kurs gehört, dass die Studierenden einen Essay schreiben, in welchem sie einen medizinischen Fall ethisch analysieren.

Kursleiterin Nikola Biller-Andorno ist Professorin für Biomedizinische Ethik an der Universität Zürich. Sie entschied sich vor zehn Jahren, die erfolgreiche Teilnahme am Kurs nicht per Multiple Choice zu prüfen, sondern ethische Fragen in der Form von Essays behandeln zu lassen. Das mache gerade im ersten Semester grossen Sinn: «Zu Beginn des Studiums ist einem das Erleben der Patientinnen und Patienten noch be- 


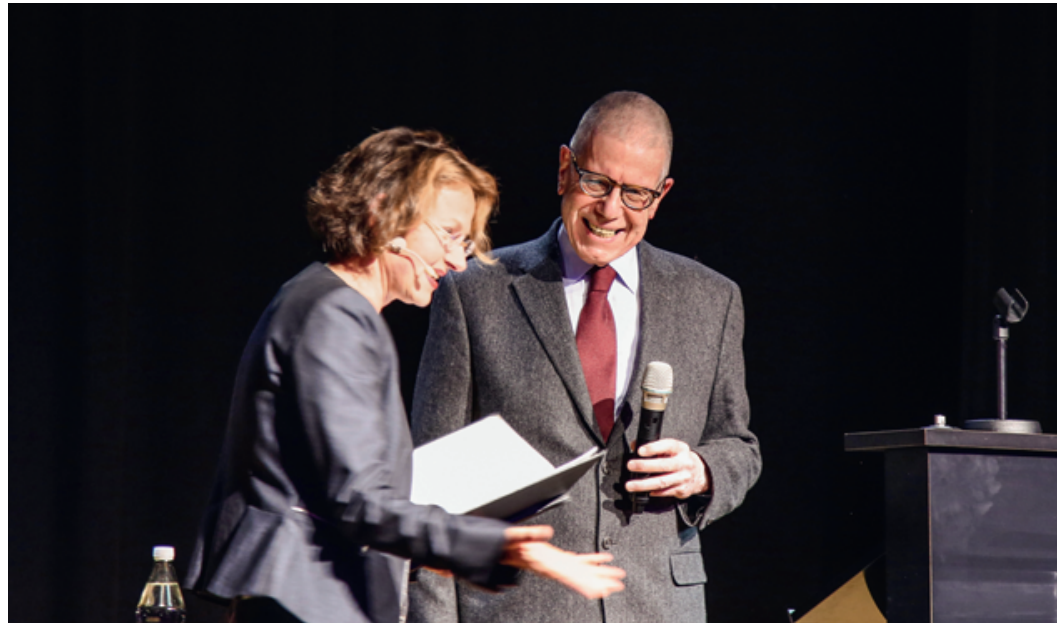

Moderatorin Hildegard Keller im Gespräch mit Preis-Stifter Edio Pusterla, Arzt und Brustkrebsspezialist.

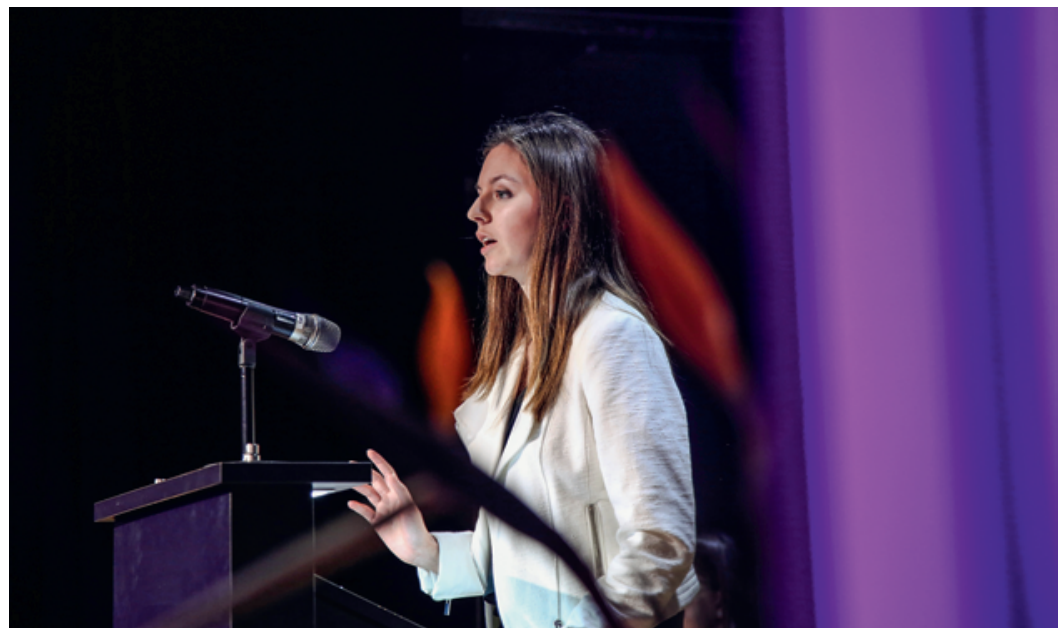

Preisträgerin Selina Steiger: Ihr Essay handelt von einem Hilfesuchenden, der eine Notfallzentrale vor ein ethisches Dilemma stellt.

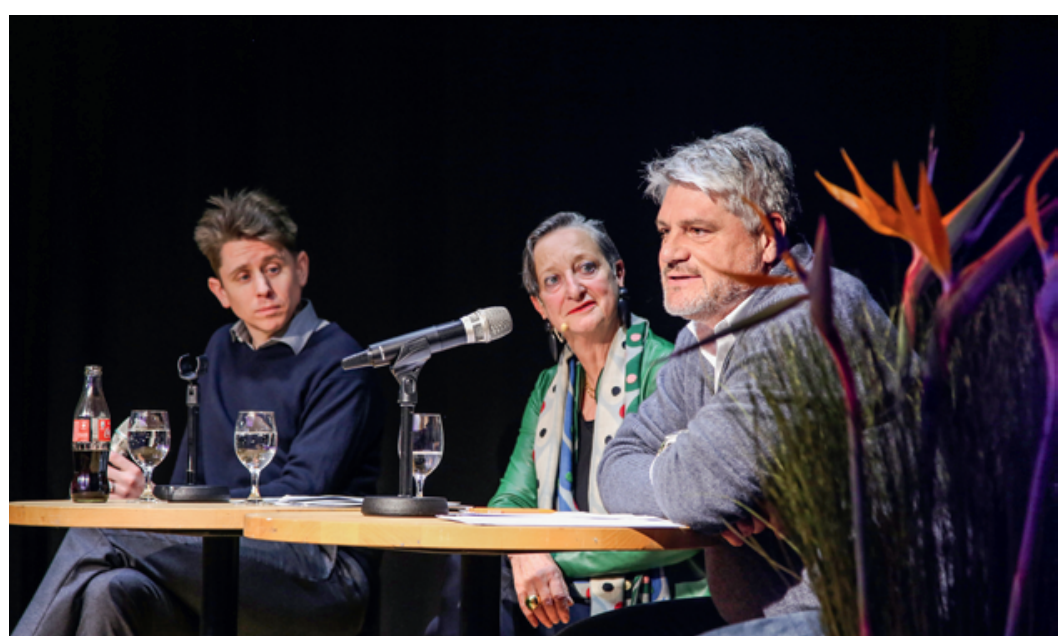

Die Jury (von links): Michael Fehr, Autor und Träger des Schweizer Literaturpreises 2018, Brida von Castelberg, ehemalige Chefärztin der Triemli-Frauenklinik, und Raoul Schrott, österreichischer Literaturwissenschaftler und Schriftsteller.

Korrespondenz:

adrianritter[at]gmx.ch sonders nah. Die Fähigkeit, eine andere Perspektive einzunehmen, sollten die Studierenden auch in ihr Arzt-Sein hineintragen", so Biller-Andorno.

In den vergangenen Jahren entstanden im Kurs zahlreiche sehr gute Texte. Deshalb möchte Biller-Andorno diese fortan einem breiteren Publikum zugänglich machen. «Ausserdem finde ich es sinnvoll, wenn es neben Preisen im Bereich der klinischen Medizin und der Grundlagenforschung auch einen Preis für Medizinethik gibt», so Biller-Andorno.

Gestiftet haben den «Premio Pusterla Junior» der Tessiner Arzt und Brustkrebs-Spezialist Edio Pusterla und die UZH-Alumni. Beteiligt am Konzept des Essay-Wettbewerbs war auch Hildegard Keller, UZH-Professorin für deutsche Literatur und Mitglied des SRF-Literaturclub-Kritikerteams. Sie unterstützte die Studierenden auch mit einem Schreibcoaching.

\section{Das Publikum entscheidet}

55 Studierende reichten einen ersten Text ein - mit einer Themenbandbreite von der Sterbehilfe bis zur ärztlichen Teilnahme an Ausschaffungsflügen von Flüchtlingen. Anfang März durften die drei Finalistinnen Gioia Epprecht, Andrina Singelmann und Selina Steiger in einem öffentlichen Finale ihre Texte vortragen. Gioia Epprecht setzt sich in ihrem Essay mit den tragischen Erlebnissen einer Migrantin im Spital auseinander. Der Text von Andrina Singelmann handelt von einer magersüchtigen Jugendlichen, die zwangseingewiesen wird. Selina Steiger beschreibt in ihrem Essay, wie ein Hilfesuchender eine Notfallzentrale vor ein ethisches Dilemma stellt (Seite 946 in dieser SÄZ).

Die Jury bestand aus der ehemaligen Chefärztin der Triemli-Frauenklinik Brida von Castelberg, dem österreichischen Literaturwissenschaftler und Schriftsteller Raoul Schrott sowie Michael Fehr, Autor und Träger des Schweizer Literaturpreises 2018.

Die Juroren nahmen eine kritische Begutachtung sowohl der ethischen Argumentation wie der literarischen Qualitäten vor - im Falle von Raoul Schrott eine bisweilen etwas gar harte Kritik. Am Schluss war sich die Jury alles andere als einig: Jede der drei Finalistinnen erhielt eine Stimme. So war es am Publikum, den Entscheid zu treffen: Selina Steiger gewann mit ihrem Text «Ein Messer, ein Feuer und viele offene Fragen - ethische Konflikte und ihre Folgen» den ersten "Premio Pusterla Junior». Der Preis soll auch in den kommenden Jahren vergeben werden. Für bereits berufstätige Ärztinnen und Ärzte soll zudem ein «Premio Pusterla Senior" geschaffen werden.

Bildnachweis

Alle Fotos: Giovanni Spitale 